\title{
Kinetics and crystallization path of a Fe-based metallic glass alloy
}

M. J. Duarte ${ }^{a, \star}$, A. Kostka ${ }^{a, b}$, D. Crespo ${ }^{c}$, J. A. Jimenez ${ }^{d}$, A.-C. Dippele, F. U. Renner ${ }^{\mathrm{a}, \mathrm{f}, \star}, \mathrm{G}$. Dehm $^{\mathrm{a}}$

\section{Affiliations:}

a Max-Planck Institut für Eisenforschung GmbH, 40237 Düsseldorf, Germany.

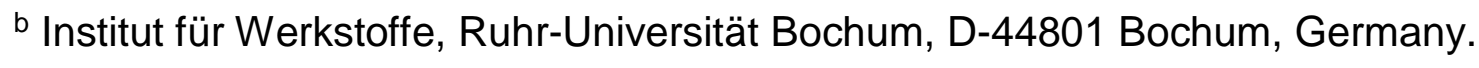

${ }^{c}$ Departament de Fisica, EETAC, Universitat Politècnica de Catalunya, 08860

Castelldefels, Spain.

d CENIM-CSIC, 28040 Madrid, Spain.

e Deusches Elektronen-Synchrotron, DESY, Hamburg, Germany.

f Instituut Voor Materiaalonderzoek, Universiteit Hasselt, 3590 Diepenbeek, Belgium.

*Correspondence to: j.duarte@mpie.de, frank.renner@uhasselt.be

\section{Abstract}

The thermal stability and the quantification of the different transformation processes involved in the overall crystallization of the $\mathrm{Fe}_{50} \mathrm{Cr}_{15} \mathrm{Mo}_{14} \mathrm{C}_{15} \mathrm{~B} 6$ amorphous alloy were investigated by several characterization techniques. Formation of various metastable and stable phases during the devitrification process in the sequence $\alpha$ - $F e, X-$ $\mathrm{Cr}_{6} \mathrm{Fe}_{18} \mathrm{Mo}_{5}, \mathrm{M}_{23}(\mathrm{C}, \mathrm{B})_{6}, \mathrm{M}_{7} \mathrm{C}_{3}, \mathrm{\eta}-\mathrm{Fe}_{3} \mathrm{Mo}_{3} \mathrm{C}$ and $\mathrm{FeMo}_{2} \mathrm{~B}_{2}$ (with $\mathrm{M}=\mathrm{Fe}, \mathrm{Cr}, \mathrm{Mo}$ ), was observed by in-situ synchrotron high energy X-ray diffraction and in-situ transmission electron microscopy. By combining these techniques with differential scanning calorimetry data, the crystallization states and their temperature range of stability under continuous heating were related with the evolution of the crystallized fraction and the phase sequence as a function of temperature, revealing structural and chemical details of the different transformation mechanisms. 


\section{Introduction}

Metallic glasses are in general acknowledged by their high mechanical strength and toughness [1-5] coupled to high corrosion resistance for some alloy compositions $[6,7]$. These unique properties provided by the amorphous structure and metallic bonding attracted increasing attention of researchers, material scientists and industries due to their great potential for basic science research and industrial applications. Examples of these are pieces of micromotors, electronic casings, cutting blades, jewelry or coatings [8-10]. Ionue et al. [11] proposed that high glass-forming ability alloys consist of at least three elements with an atomic size mismatch above $12 \%$ between them and negative heats of mixing among the three main elements. However, high material costs associated to the price of the elemental additions which lead to easiest glass formation (e.g. Ln, Ga, Zr, Hf, Nb, Pd, Pr, Y) [12] limit the wide commercial acceptance of most alloy systems. The main market is still concentrated in Fe-based metallic glasses using abundant and inexpensive elements such as B, C, Si and P [13]. Their soft magnetic behavior and high saturation magnetization allow fabricating soft magnetic cores for high efficiency transformers, magnetic heads or electronic article surveillance items [9] that account for the most widespread applications. However, Febased alloys are also produced for shot peening particles [14] or corrosion and wear resistant coatings over steel substrates [9] due to their additional wear and corrosion resistance.

As metastable alloys, properties' modification eventually occurs through crystallization. Some characteristics of metallic glasses can be superior to their crystalline counterparts $[7,15,16]$, and adverse properties can be mitigated by developing composites with ductile or hard crystals in a bulk amorphous matrix [17-19] or a twophase nanocomposite microstructure [20-24]. Hence, the knowledge of the crystallization kinetics and the phase sequence is essential to either determine the 
thermal stability of the alloy under specific conditions or to optimize the generation route of new microstructures.

We studied here the base amorphous alloy $\mathrm{Fe}_{50} \mathrm{Cr}_{15} \mathrm{Mo}_{14} \mathrm{C}_{15} \mathrm{~B}_{6}$ [25], to which noble metals or rare earth elements are added to increment the glass forming ability [26-27]. High concentrations of $\mathrm{Cr}$ provide corrosion and oxidation resistance [28], while about 20 at. \% of non-metals $(C, B, P, S, N)$ are required to maintain the amorphous structure during cooling [13]. Subsequent property improvements are related to Mo, which reduces the intensity of the oxidizing effect required to ensure passivity and decreases the tendency of previously formed passive films to break down [29]. The addition of these elements to Fe-based alloys promotes a solid solution strengthening effect [30] and drives the precipitation of homogeneously dispersed second phase particles, such as carbides or borides $[16,30]$.

In our previous reports $[7,16]$ we correlated the corrosion behavior to the increasing crystalline character of the alloy, while focusing on the chemical changes. The goal of the current study is to present a thorough analysis on the crystallization path and kinetics of the $\mathrm{Fe}_{50} \mathrm{Cr}_{15} \mathrm{Mo}_{14} \mathrm{C}_{15} \mathrm{~B}_{6}$ amorphous alloy, determine the temperature ranges where different phases are stable, and discuss the mechanisms by which each one of the different transformations occur. The analysis is based on the advantage of in-situ transmission electron microscopy (TEM) and in-situ synchrotron-radiation X-ray diffraction (SR-XRD) techniques allowing imaging and microstructural characterization while heating up the amorphous sample; the thermodynamic properties are measured by differential scanning calorimetry (DSC). The challenge for the analysis resides in the multicomponent microstructure developed and the very close interplanar distances of the different atomic packings, making difficult an unambiguous assessment of the single phases formed throughout crystallization. 


\section{Experimental}

Ribbons of the $\mathrm{Fe}_{50} \mathrm{Cr}_{15} \mathrm{Mo}_{14} \mathrm{C}_{15} \mathrm{~B}_{6}$ amorphous alloy (30-40 $\mu \mathrm{m}$ thickness) were prepared by induction melting a master alloy in a boron nitride crucible, further ejected over a rotating copper wheel at a speed of $40 \mathrm{~m} / \mathrm{s}$. The used master alloy was produced by arc melting the individual elements, of purity higher than $99.9 \%$, in a Ti-gettered argon atmosphere.

In-situ XRD measurements using high-energy SR-XRD were performed at the P02.1 beamline from the PETRA III storage ring at DESY, Hamburg [31]. The samples were mounted by copper clamps in a custom made resistive heating holder at a sample-todetector distance of $440 \mathrm{~mm}$. An argon atmosphere was provided by the gas flow in a region confined by Kapton ${ }^{\circledR}$ windows. The current was increased manually following a step ramp of $\sim 0.05 \mathrm{~A} / \mathrm{min}$. The samples were measured in the transmission mode with

a monochromatic beam of $59.63 \mathrm{keV}(0.2079 \AA)$. The diffraction patterns were collected on a two-dimensional detector (PerkinElmer XRD 1621) and integrated by using the Fit2D software [32]. The measured standard reference was LaB6. XRD data refinement was performed with the Rietveld analysis program TOPAS (Bruker AXS), following the approach given in [16].

Thin lamellae were extracted from the ribbons by focused ion beam (FEI Helios Nanolab $^{\text {TM }} 600 \mathrm{i}$ dual-beam operated at $30 \mathrm{kV}$ ) to perform in-situ heating TEM measurements. The procedure was a modification to the method proposed in [33]. Before thinning, an additional support was left on the sample base and lateral regions to withstand the internal stresses generated during crystallization and avoid rolling of the lamella. Once thinned, the sample was lifted-out by using a micromanipulator that allowed axial rotation of the sample for its placement over a MEMS-heating chip [34]. The existing SiN window of the chip was milled out to provide a clean image of the sample region. Finally, the sample surface was cleaned with low energy ions (5 keV) 
to remove the possible platinum spread generated during the sample welding over the chip.

In-situ TEM measurements were carried out in a Jeol JEM-2200FS operating at 200 $\mathrm{kV}$ using the single tilt heating holder from DENS-Solutions. Different heating strategies regarding temperature rate and holding times were used to observe the crystallization behavior and these are further described within the next sections. An increase of the experiment temperature results in a small change of the specimen height-position (the eucentric height) with respect to the back focal plane of the microscope. Thus, before acquisition of every electron diffraction pattern the eucentric height of the sample and objective focus were aligned. For clarity, the colors were inverted in all reported diffraction patterns.

Structural information about the forming phases was derived using different methods; first, by analyzing the fast Fourier transform (FFT) of high-resolution TEM (HR-TEM) images for large crystals; second, by measuring interplanar distances on selected area electron diffraction patterns (see Section 3.2); third, by analyzing rotationally averaged patterns when the number and distribution of the diffracted spots did not allow for unambiguous measurements. Finally, spot diffraction pattern simulations were performed using JEMS software [35] for the largest crystals found. The crystal references used in this study were taken from the inorganic crystal structure database (ICSD): Fe_alpha_icsd_53802, FeCrMo_icsd_625901, M23C6_icsd_62671, Cr7C3_icsd_181713, Fe2O3_icsd_184766, FeMo2B2_icsd_44292. For the detailed analyses of the diffraction patterns, the measured interplanar distances were compared to the reference data and small positive deviations were considered, indicating a lattice expansion. As many signals stemming from different phases overlap with each other, the individual phases were included one by one in the analysis. A new 
phase was therefore introduced only when the already present phases could not account for all the measured signals.

The kinetics of the crystallization process was investigated using a heat flux type Netzsch 404F3 DSC equipment. The baseline correction was applied by measuring an empty sample pan for each run. The excess apparent specific heat $m(T)$ used in the developed model was measured as a function of the temperature in a continuous ramp at a heating rate of $20 \mathrm{~K} / \mathrm{min}$. The crystallization steps, enthalpy of the transformations and crystallized volume fraction were determined from the exothermic peaks observed on the continuous heating curve. In addition, the activation energies of the different transformations were estimated from measurements at heating rates of 5, 10, 15, 20 and $25 \mathrm{~K} / \mathrm{min}$.

\section{Results}

\subsection{In-situ high energy synchrotron X-ray diffraction}

SR-XRD patterns obtained sequentially during heating were used to perform in-situ studies of the phase evolution sequence during the devitrification process. Fig. 1 shows one-dimensional XRD profiles obtained by integrating 2-D Debye-Scherrer rings patterns collected during heating. Rapid identification of the crystalline species using the JCPDS database allowed to determine the presence of six phases through the whole current range: $\alpha-\mathrm{Fe}, \chi-\mathrm{Cr}_{6} \mathrm{Fe}_{18} \mathrm{Mo}_{5}, \mathrm{M}_{7} \mathrm{C}_{3}, \mathrm{M}_{23}(\mathrm{C}, \mathrm{B})_{6}, \mathrm{M}_{6} \mathrm{C}\left(\eta-\mathrm{Fe}_{3} \mathrm{Mo}_{3} \mathrm{C}\right)$ and FeMo2B 2 , with $\mathrm{M}=\mathrm{Fe}, \mathrm{Cr}$, Mo.

Matching the XRD pattern with the JCPDS database did not allow for the unequivocal characterization of the phases present due to systematic peak overlap. Rietveld refinement facilitates a more complete and reliable interpretation of the experimental data since this method fits the whole diffraction pattern and all reflections, overlapping or not. Besides, the Rietveld analysis allows determining the proportion of each crystalline and amorphous phase, and simultaneously extracts information about the 
crystallite size and lattice parameters. Fig. 2 and Fig. 3 show the results of the Rietveld refinement as a function of the applied current, obtained from the first diffraction rings collected immediately after a current increase. In Fig. 2, stepped increments observed throughout the applied current vs. time curve (black solid line) are related to the manual increase of the current used for heating the sample, and thus, are responsible for the observed jumps throughout the crystallization behavior.

Crystallization starts at $1.95 \mathrm{~A}$ with the nucleation of small grains of $\alpha-\mathrm{Fe}(<5 \mathrm{~nm}$, as shown in Fig. 2) and larger $\chi-\mathrm{Cr}_{6} \mathrm{Fe}_{18} \mathrm{Mo}_{5}$ crystals. This result confirms and strengthens our preliminary observation of the $\alpha$-phase by TEM and APT analyses [16]. The $\alpha$ phase was only detected at $1.96 \mathrm{~A}$ in a fraction of about $3 \%$. In a further step, at 1.98 A, a mixed structure of crystalline $\chi-\mathrm{Cr}_{6} \mathrm{Fe}_{18} \mathrm{Mo}_{5}, \mathrm{M}_{23}(\mathrm{C}, \mathrm{B})_{6}, \mathrm{M}_{7} \mathrm{C}_{3}$ and amorphous phases was observed. Mo-rich phases appeared at higher currents: $\eta-\mathrm{Fe}_{3} \mathrm{Mo}_{3} \mathrm{C}$ at 2.14 $\mathrm{A}$ and $\mathrm{FeMo}_{2} \mathrm{~B}_{2}$ at $2.69 \mathrm{~A}$. Crystallization is completed at $2.40 \mathrm{~A}$, indicated by the white dashed line in Fig. 1, and therefore the last transformation (precipitation of $\mathrm{FeMo}_{2} \mathrm{~B}_{2}$ ) occurs within one of the already formed phases. The initially transformed phases $\alpha, \chi$ and $\mathrm{M}_{7} \mathrm{C}_{3}$ are non-equilibrium and they dissolve into the more stable carbides $\mathrm{M}_{23}(\mathrm{C}, \mathrm{B})_{6}$ and $\eta-\mathrm{Fe}_{3} \mathrm{Mo}_{3} \mathrm{C}$. The ratio of the crystalline fraction between these two last phases remains constant at 3:1 (from Fig. 2).

As shown in Fig. 3, the changes in the lattice parameters depict different slopes with the increase of the applied current. The observed trends are partially in agreement with the different ascents in the crystal growth. Direct estimations on the crystallization kinetics derived from these measurements are not possible due to the inherent inaccuracies related to the experimental conditions, and especially the step ramp followed during heating. However, qualitative assessments related to diffusional processes can be extracted and are discussed in section 4 . 


\subsection{In-situ transmission electron microscopy}

Figure 4 shows bright field TEM images (BF-TEM) and selected area electron diffraction (SAD) patterns obtained for as-spun ribbons and at the initial stage of crystallization. The white dashed circles represent the selected area aperture applied for collecting the diffraction data. The initial amorphous structure of the alloy (bright field image in Fig. 4a) was confirmed by a broad reflection in the SAD pattern (Fig. 4b). After heating up to $713 \mathrm{~K}$ in a ramp of $20 \mathrm{~K} / \mathrm{s}$ and subsequent cooling down to room temperature (RT), crystallites with an average size of $21 \pm 5 \mathrm{~nm}$ embedded in the amorphous matrix are observed (Fig. 4c). The crystallites highlighted by arrows pointing down in the BF image were selected for HR-TEM investigations, as illustrated in Fig. 4e-f. Both, the measured interplanar distances from the diffraction pattern of Fig. $4 \mathrm{~d}$ and the FFT of Fig. $4 \mathrm{f}$ (given in the top right corner of the corresponding figures) allow identifying these crystals as $\chi-\mathrm{Cr}_{6} \mathrm{Fe}_{18} \mathrm{Mo5}$. All further raw diffraction data and corresponding identified phases are given in Table A1. The diffracted spots at this initial state cannot be resolved by radial intensity profiles of the rotationally averaged diffraction patterns as they get obscured by the broad reflection of the amorphous phase (Fig. A1).

Once the first formed crystallites were identified, a second heating ramp at $20 \mathrm{~K} / \mathrm{s}$ was applied up to $723 \mathrm{~K}$ and held during $1 \mathrm{hr}$ for isothermal annealing. The microstructural changes were monitored by BF imaging and SAD (Fig. 5) in the same region of interest, indicated by white arrows in Fig. 5a-c. Although fast crystal nucleation of $\chi-\mathrm{Cr}_{6} \mathrm{Fe}_{18} \mathrm{Mo}_{5}$ can be detected initially in the considered region, a second phase, identified as $M_{23}(B, C)_{6}$, is only observed after annealing for more than $100 \mathrm{~s}$. In addition to the analysis of the rotational average of the radial intensity profile of the corresponding diffraction pattern of Fig. 5g, independent measurements of the diffracted spots were 
taken manually, as the brightness of the broad reflection of the amorphous phase still hinders the identification of such signals in Fig. 5j. A third phase forms after annealing for 15 min (Fig. 5e,h), recognized as $\mathrm{M}_{7} \mathrm{C}_{3}$ in Fig. 5k. Annealing up to $1 \mathrm{hr}$ caused only crystal growth of these three phases (Fig. 5f). Nevertheless, the observed amorphous ring remained strong (Fig. 5i) and no signals of a new phase appeared (Fig. 5l).

Formation of oxides during in-situ TEM was observed at $723 \mathrm{~K}$ (Fig. 6). The first trace of their presence appears after annealing for $30 \mathrm{~s}$ as dark diffuse regions (Fig. 5c). Full identification can be only achieved when these oxides become large enough to assess their crystalline nature by HR-TEM or SAD. Fig. 6 a shows an overview of a larger area of the sample after annealing for $20 \mathrm{~min}$. A SAD pattern from one of those large dark regions is presented in Fig. 6b. Although overlapping of the different amorphous and crystallized phases is observed, the strongest and regular signals correspond to hematite, $\mathrm{Fe}_{2} \mathrm{O}_{3}$, as indicated by the overlay of the measured data and the simulated diffraction of this oxide in Fig. 6c. Oxide formation upon annealing is not surprising due to the amount of oxygen present in the TEM column (pressure $\sim 2.5 \times 10^{-5}$ mbar) and the oxygen gathered during the TEM sample storage or transfer times from FIB or TEM. These conditions are enough to generate a few monolayers of oxygen over the sample surface. Surface diffusion of oxygen is further enhanced with temperature favoring the formation of oxides that are easily perceived while examining the small volumes probed here by TEM.

Annealing in steps of $50 \mathrm{~K}$ from 723 to $973 \mathrm{~K}$ and holding times of 5 min was finally performed to determine the additional phases formed during crystallization (Fig. 7). Diffraction spots corresponding to $\eta$ - $\mathrm{Fe}_{3} \mathrm{Mo}_{3} \mathrm{C}$ were identified at $773 \mathrm{~K}$ and full crystallization was observed above $873 \mathrm{~K}$ in the approach followed here. While $\mathrm{M}_{7} \mathrm{C}_{3}$ is still identified at $873 \mathrm{~K}$, the specific signals of the $\chi-\mathrm{Cr}_{6} \mathrm{Fe}_{18} \mathrm{Mo}_{5}$ cannot be confirmed, indicating its full transformation into a more stable phase. HR-TEM and SAD patterns 
were acquired containing information mainly of individual large crystals (as the region enclosed by the white dashed circle) after annealing the microstructure at $973 \mathrm{~K}$ (Fig. $7 \mathrm{~d}-\mathrm{f})$. The interplanar distances obtained by FFT of the HR-TEM images and simulation patterns of the diffraction data confirmed the final microstructure as a dual phase $\mathrm{M}_{23}(\mathrm{~B}, \mathrm{C})_{6}$ and $\eta-\mathrm{Fe}_{3} \mathrm{Mo}_{3} \mathrm{C}$. Unfortunately, annealing at higher temperatures to observe the transformation of $\mathrm{FeMo}_{2} \mathrm{~B}_{2}$ was limited by the setup and sample characteristics.

\subsection{Crystallization kinetics}

Crystallization kinetics was studied by DSC measurements of the excess apparent specific heat, $m(T)$, at a heating rate of $20 \mathrm{~K} / \mathrm{min}$. Four exothermic transformations are observed in Fig. 8a with peak temperatures of 891, 927, 976 and $999 \mathrm{~K}$, which will be labelled $\alpha_{1}-\chi-\mathrm{Cr}_{6} \mathrm{Fe}_{18} \mathrm{Mo}_{5}-, \alpha_{2}-\mathrm{M}_{23}(\mathrm{C}, \mathrm{B})_{6}-, \alpha_{3}-\mathrm{M}_{7} \mathrm{C}_{3}-$ and $\alpha_{4}-\mathrm{M}_{6} \mathrm{C}\left(\eta-\mathrm{Fe}_{3} \mathrm{Mo}_{3} \mathrm{C}\right)$ -. According to the previous observations, the first crystalline phase, $\alpha-\mathrm{Fe}$, is too faint to produce a distinct exothermic peak, and the last transformation, corresponding to the precipitation of $\mathrm{FeMo}_{2} \mathrm{~B}_{2}$ as detected by $\mathrm{XRD}$, occurs at a peak temperature of $1159 \mathrm{~K}$ and is not included in the model. TEM observations reported above show that the sample is fully crystallized while the $\mathrm{Fe}_{3} \mathrm{Mo}_{3} \mathrm{C}$ phase is forming; thus, the transformation $\alpha_{4}$ is a secondary precipitation, and must be modelled accordingly. The determined specific heat $c(T)$ is shown in Fig. 8a. Each transformation contributes to the total transformation enthalpy $h(T)$ according to its specific enthalpy. No independent measurements of these specific enthalpies were available, and as a first approximation we assumed that all of them are equal. Then, the total enthalpy is just the addition of that of each transformation:

$$
h(T)=h_{1}(T)+h_{2}(T)+h_{3}(T)+h_{4}(T)
$$

This allows defining the corresponding transformed fractions as

$$
x_{i}(T)=\frac{h_{i}(T)}{h_{i}\left(T_{e}\right)}
$$


To determine the enthalpy of the transformation the specific heat of the alloy, $c(T)$, must be subtracted. At the beginning of the transformation $\left(T_{s}\right)$ the specific heat is that of the undercooled liquid, $c_{l}$, and at the end of the crystallization it is that of the solid, $c_{s}$, considered the same that at $T_{e}$. We assume that along the three initial crystallizations the already transformed phases contribute to the total specific heat with the specific heat of the solid, while the remaining untransformed volume contributes with the specific heat of the liquid,

$$
c(T)=c_{l}+\left(c_{s}-c_{l}\right) \cdot x_{p}(T)
$$

where $x_{p}(T)$ is the total primary transformed fraction at temperature $T$, defined as

$$
x_{p}(T)=\gamma_{1} x_{1}(T)+\gamma_{2} x_{2}(T)+\gamma_{3} x_{3}(T)
$$

where $\gamma_{1}, \gamma_{2}$ and $\gamma_{3}$ are the total volume fraction of the first three crystallizations at the end of each of them, respectively. Thus, the alloy specific heat and the transformed fraction are interrelated; however, they can be determined simultaneously along the fitting. The alloy specific heat is considered to have a linear dependence in $T$ to initialize the fitting procedure,

$$
c(T)=c\left(T_{s}\right)+\frac{c\left(T_{e}\right)-c\left(T_{S}\right)}{T_{e}-T_{S}}\left(T-T_{S}\right) .
$$

This estimated alloy specific heat is used to obtain a first approximation to the total enthalpy of the transformation $h(T)$,

$$
h(T)=\int_{T_{S}}^{T}[m(T)-c(T)] d T
$$

and successive refinements of the transformed fraction are reflected into the specific heat through equation (3). 
The individual transformations are modeled in the Kolmogorov-Johnson \& MehlAvrami (KJMA) framework [36]. Isothermal transformations are usually well modeled with the isothermal KJMA expression

$$
x(t)=1-\exp \left(-k \cdot t^{n}\right)
$$

where $k$ is the kinetic constant and $n$ is the so-called Avrami exponent. Differentiating this equation yields the KJMA rate equation [37]

$$
\frac{d x(t)}{d t}=n \cdot k \cdot[1-x(t)] \cdot[-\ln (1-x(t))]^{(n-1) / n}
$$

which can be used to model non-isothermal transformations. The growing phases can be described as grains with a nucleation rate $N(T)$ and a growth rate $G(T)$ following an Arrhenius dependence,

$$
N(T)=N_{0} \cdot \exp \left(-\frac{E_{N}}{k_{B} T}\right), \quad G(T)=G_{0} \cdot \exp \left(-\frac{E_{G}}{k_{B} T}\right)
$$

where $k_{B}$ is the Boltzmann constant. In the isokinetic approximation $E_{N}=E_{G}=E$ and the kinetic constant has then the same temperature dependence

$$
k(T)=k_{0} \cdot \exp \left(-\frac{E}{k_{B} T}\right) .
$$

Consequently, the enthalpy released along the transformation depends on $k, n$ and $E$. Bhadeshia showed that this model can be extended to simultaneous transformations [38]. This approach is valid for transformations filling the space, as it happens with the third and maybe the fourth transformations here. Here transformations are successive, as primary transformations do not fill all the space. As the nanocrystalline isolated particles precipitated within the amorphous matrix have a chemical composition different than the untransformed phase, these primary transformations are diffusion controlled. The growth rate, in this case, is radius dependent, controlled by the diffusion coefficient, i.e. 


$$
G(T)=\frac{D(T)}{2 r}
$$

$r$ being the radius of the crystallite. The transformed phases are stabilized by specific elements, and thus, their nucleation and growth rate are related to the diffusion of these atoms from the amorphous matrix. Thus, the untransformed phase will be depleted in these species and eventually the growth velocities of the crystalline phases, and perhaps also the nucleation rate, will be reduced due to lack of the required composition. This mechanism is known as diffusion controlled growth with soft impingement. This implies that the amorphous fraction is transformed more slowly at the end of the reaction, and the DSC signal shows a longer tail at higher temperatures than for purely diffusion controlled growth $[39,40]$. Furthermore, this growth mechanism effectively ends the transformation before filling the whole space. Initially, this behavior was attributed to a decreasing Avrami exponent. However, Pradell et al. [39] showed that primary transformations can be modelled by assuming a concentration dependent diffusion coefficient. In transformation $\alpha_{1}$, the diffusion coefficient would read

$$
D_{1}\left[T, x_{1}(T)\right]=D_{1}(T) \frac{1-x_{1}(T)}{1-\gamma_{1} x_{1}(T)}
$$

where the diffusion coefficient is multiplied by the ratio between the untransformed primary phase and the available space for growth as the transformation proceeds. In transformation $\alpha_{2}$, this correction reads

$$
D_{2}\left[T, x_{2}(T)\right]=D_{2}(T) \frac{1-x_{2}(T)}{1-\gamma_{1} x_{1}(T)-\gamma_{2} x_{2}(T)}
$$

Here the denominator is modified to include the space already transformed by $\alpha_{1}$. This approach allowed to recover the original sense of the Avrami exponent, which now remains constant along the transformation as the decrease in the transformation rate is due to the concentration dependent diffusion coefficient. 
For the temperature dependence of the diffusion coefficient we assumed also an Arrhenius dependence. As a consequence, our model is written as follows

$$
\begin{gathered}
k_{1}\left(T, x_{1}\right)=k_{01} \cdot \exp \left(-\frac{E_{1}}{k_{B} T} \frac{\left[1-x_{1}(T)\right]}{\left[1-\gamma_{1} x_{1}(T)\right]}\right) \\
k_{2}\left(T, x_{1}, x_{2}\right)=k_{02} \cdot \exp \left(-\frac{E_{2}}{k_{B} T} \frac{\left[1-x_{2}(T)\right]}{\left[1-\gamma_{1} x_{1}(T)-\gamma_{2} x_{2}(T)\right]}\right) \\
k_{3}(T)=k_{03} \cdot \exp \left(-\frac{E_{3}}{k_{B} T}\right) \\
k_{4}(T)=k_{04} \cdot \exp \left(-\frac{E_{4}}{k_{B} T}\right)
\end{gathered}
$$

However, the activation energies can be determined by the Kissinger method (see supplementary material and Fig. A2). For computing simplicity the model is written so as each transformation starts at a given temperature $T_{0 i}$. Additionally, the long tail of the primary crystallizations may delay them even after the third crystallization has finished. This fact lacks of physical sense, as crystallization $\alpha_{3}$ exhausts the space. For this reason, the rate equation (8) is corrected for $\alpha_{1}$ and $\alpha_{2}$ by a factor $\left[1-x_{3}(t)\right]$. The rate equation for $\alpha_{1}$ and $\alpha_{2}$ with this correction and including the soft impingement factor becomes

$$
\frac{d x_{1,2}}{d t}=\left[1-x_{3}\right] \cdot \frac{\emptyset_{1,2} t^{n-1} k_{1,2}\left(n_{1,2}+t \frac{E_{1,2}}{k_{B} T^{2}} \frac{d T}{d t}\right)\left(1-x_{1,2}\right)}{1-k_{1,2} t^{n} \frac{d \emptyset_{1,2}}{d x_{1,2}}\left(1-x_{1,2}\right)}
$$

Here, the specific functional dependencies are removed for the sake of clarity, that is $\varnothing(x(t)) \equiv \emptyset$ and so on. The full derivation of equation (14) and numerical integration is given in the supplementary materials.

Also for $\alpha_{1}$ and $\alpha_{2}$, primary crystallizations, the Avrami exponents are physically constrained to remain between 1.5 and 2.5, values which, according to the literature, correspond to diffusion controlled growth with pre-existing nuclei or constant nucleation respectively. Thus, the model depends on four parameters per transformation, namely 
$k_{0 i}, n_{i}, T_{0 i}$ and $\gamma_{i}$ for each transformation, with the restrictions $\gamma_{1}+\gamma_{2}+\gamma_{3}=1$ and $1.5 \leq n_{1,2} \leq 2.5$, for a total of 15 fitting parameters. This is the minimum number of free parameters, given that:

- The Avrami model has three free parameters for temperature dependent transformations, but the activation energies are determined by the Kissinger method (8 free parameters).

- The diffusion controlled growth model adds a third parameter per transformation, which corresponds to the transformed volume fraction at the end of the primary transformation. The third transformation can only fit the untransformed space, and this is the origin of the first restriction (2 free parameters).

- The calorimetric signal of the fourth transformation must be scaled against the previous three; this is the origin of $\gamma_{4}$ (1 free parameter).

- Each transformation starts at a different temperature (4 free parameters).

The fit of the specific heat is shown in Fig. 8a. The fitted enthalpy is plotted in Fig. $8 \mathrm{~b}$ and compared to the measured enthalpy. Though the fit of the specific heat is not perfect, the fitted enthalpy is very close to the experimental data.

Table 1 shows the values of the fitted parameters after fitting the transformed fraction. The values of the Avrami exponents can be interpreted according to the literature [41]. $\alpha_{1}$ and $\alpha_{2}$ show Avrami exponents of 2.5, the upper limit for diffusion controlled grain growth; in this case, it corresponds to continuous nucleation. $\alpha_{3}$ has a very high value of 6.3 related to two factors. In the one hand, an Avrami exponent above 4 is usually associated to a non-constant - i.e., increasing - nucleation [42] and/or non-random nucleation, as for example new born crystallites appearing at the boundary of previously developed grains. As transformation $\alpha_{3}$ develops after two consecutive primary crystallizations it is feasible that $\alpha_{3}$, the final crystallization of the remaining amorphous matrix, may develop very fast, with a highly heterogeneous distribution of 
crystallization nuclei, leading to a high value of the Avrami exponent. However, it is worth to mention that as the $\alpha_{3}$ develops on the tail of the two previous primary crystallizations, the modeling of the tail affects strongly the value of $n_{3}$. Thus, the uncertainty on $n_{3}$ is estimated to be high, and the true value of $n_{3}$ might be lower than that obtained from the fit. As for $n_{4}$, the value of 3.6 may correspond to a crystallization process with interface controlled growth. Such processes show Avrami exponents between 3, pre-existing nuclei, and 4, constant nucleation. Once more, as $\alpha_{4}$ develops on the tail of $\alpha_{3}$, the uncertainty in $n_{3}$ is also affecting $n_{4}$. Visual inspection of Fig. $8 \mathrm{a}$ shows poor quality of the fit between 985 and $1000 \mathrm{~K}$. Thus, we consider that $n_{4}$ might be slightly underestimated, and $\alpha_{4}$ is likely a transformation with interface controlled growth and constant nucleation.

\section{Discussion}

The initial crystallization event $\alpha_{1}$ of the $\mathrm{Fe}_{50} \mathrm{Cr}_{15} \mathrm{Mo}_{14} \mathrm{C}_{15} \mathrm{~B}_{6}$ amorphous alloy involves the simultaneous nucleation of two crystalline phases embedded in the amorphous matrix: a bcc solid solution ( $\alpha$ ) and the intermetallic Fe-Cr-Mo $\chi$-phase. The $\chi$-phase has an $\alpha-M n$ structure with an unit cell consisting of 58 atoms distributed on 4 sublattices with $2,8,24$, and 24 atoms on each that differentiates it from other closepacked structures $[43,44]$. $\alpha$-Fe is stable only for a short temperature range and forms in a small amount (less than $5 \%$ ) with crystallite size below $5 \mathrm{~nm}$, as shown by the present Rietveld refinement of the XRD data (Fig. 2). As the XRD peak positions of $\alpha$ and $\chi$-phases match closely and the diffraction lines are very broad due to the small crystallite size for both phases, it can be difficult to identify the phase using XRD, explaining why a-Fe was not observed in our previous investigations [16]. TEM and DSC analyses performed in this work were neither conclusive in the detection of this phase due to overlapping signals with the intermetallic $\chi$-phase. However, Fe-rich structures of the form $\alpha-F e$ and $\alpha$ '-Fe have been reported during crystallization of other 
Fe-Cr-Mo-C amorphous alloys [30] and its presence was already suggested in [16], and were therefore considered in the present Rietveld refinement. Furthermore, this transient phase might be responsible for the slight mismatch between the experimental and fitted specific heat at the beginning of the transformation (869 to $876 \mathrm{~K}$ ). As for the kinetics of the transformation, the model of diffusion controlled growth with soft impingement and constant nucleation fits the measured specific heat with notable accuracy.

Carbide and carboboride transformations follow the crystallization process due to the high $\mathrm{C}$ and $\mathrm{B}$ content in the alloy. The initial formation of the large bcc phases causes enrichment of $\mathrm{C}$ and $\mathrm{B}$ within the amorphous matrix, leading the primary nucleation of a complex fcc $\mathrm{M}_{23}(\mathrm{C}, \mathrm{B})_{6}$ phase. Carbides of the form $\mathrm{M}_{23} \mathrm{C}_{6}$ are among the most common Cr-rich carbides in steels. The $\mathrm{M}_{23} \mathrm{C}_{6}$ preferential nucleation at lower temperatures with respect to other carbide phases is related to its relative low enthalpy of formation when no other strong carbide formers such as $\mathrm{Ti}$ or $\mathrm{V}$ are present in the alloy. $M_{23}(C, B)_{6}$ is the predominant phase in the crystallized sample during the present process, accounting up to $70 \%$ of the crystallized fraction. This transformation is associated to the second exothermic peak in the DSC data. The kinetics is well resolved by the primary transformation model with constant nucleation, showing a good agreement with the experimentally determined specific heat.

The presence of the $M_{7} C_{3}$ phase is confirmed here as opposite to the reported $M_{3}(B, C)$ borocarbide. Misinterpretation of the orthorhombic phase as $\mathrm{M}_{3}(B, C)$ could be due to a high background and low counts obtained previously by conventional XRD. This problem was overcome here by the use of synchrotron radiation. TEM and former APT measurements [16] show that the compositions of the untransformed and transformed phases during the $\alpha_{3}$ crystallization are the same. According to the literature, the Avrami exponent for an interface controlled growth with constant nucleation rate is 4 . 
However, the Avrami exponent $n_{3}$ shows in this case a larger value, probably due to increasing and/or non-random nucleation.

The $\alpha_{4}$ transformation takes place therefore in an already crystalized material. The initially transformed phases $\alpha, \chi$ and $\mathrm{M}_{7} \mathrm{C}_{3}$ are metastable and they dissolve into the more stable carbides $\eta-\mathrm{Fe}_{3} \mathrm{Mo}_{3} \mathrm{C}$ and $\mathrm{M}_{23}(\mathrm{C}, \mathrm{B})_{6} . \alpha_{4}$ corresponds to the $\chi-\mathrm{Cr}_{6} \mathrm{Fe}_{18} \mathrm{Mo}_{5}$ to $\eta-\mathrm{Fe}_{3} \mathrm{Mo}_{3} \mathrm{C}$ transformation while $\mathrm{M}_{7} \mathrm{C}_{3}$ slowly dissolves into $\mathrm{M}_{23}(\mathrm{C}, \mathrm{B})_{6}$, however enriching with Mo the newly formed $\eta-\mathrm{Fe}_{3} \mathrm{Mo}_{3} \mathrm{C}$ phase. The value of the Avrami exponent $n_{4}$ is 3.6 , but due to the fitting uncertainty between 985 and $1000 \mathrm{~K}$ it might be slightly underestimated. According to the literature, a transformation with interface controlled growth and pre-existing nuclei shows an Avrami exponent of 3 . Thus, the more probable description of the fourth transformation is that those regions with the proper composition transform into the new phase while the remaining phases continue without changes until the fifth transformation, not considered in this analysis, that takes place at about $1159 \mathrm{~K}$.

The evolution of the chemical composition is partially accounted in the analysis of the lattice parameters. From the XRD data in Fig. 3, a linear increment on the lattice parameters with temperature should be observed if the variations are attributed solely to thermal expansion. However, here the chemical composition change of the different phases by atomic diffusion must be also considered to explain the different tendencies followed by the independent phases observed in Fig. 3. For instance, the slight decrease on ao for $\mathrm{M}_{23}(\mathrm{C}, \mathrm{B})_{6}$ upon heating by up to $2.45 \mathrm{~A}$ can be related to the continuous rejection of Mo atoms from the formed crystals [16]. The further linear increase on ao up to $2.69 \mathrm{~A}$ is then associated to mainly thermal dilatation with a small or no compositional change. At the latter current, the formation of the Mo-rich $\mathrm{FeMo}_{2} \mathrm{~B}_{2}$ requires diffusion of Mo away from $\mathrm{M}_{23}(\mathrm{C}, \mathrm{B})_{6}$ causing a sudden decrease in the lattice parameters up to $2.73 \mathrm{~A}$. Finally, the two different positive slopes perceived above this 
current indicate the close competition of both processes. A similar behavior is observed for $\eta-\mathrm{Fe}_{3} \mathrm{Mo}_{3} \mathrm{C}$. The initial fast slope up to a current of $2.33 \mathrm{~A}$ is related to the incorporation of the big Mo atoms [16], followed by a more stable linear trend related to thermal effects up to the formation of $\mathrm{FeMo}_{2} \mathrm{~B}_{2}$. At this point, diffusional processes take a prominent role in the three cohabiting phases up to the chemical stabilization above $3 \mathrm{~A}$.

As per the activation energies, they decrease in the three first transformations, but increase again in the fourth one. This may also support the hypothesis that the forth transformation takes place in an already transformed phase.

Finally, the formation at high temperatures of $\mathrm{FeMo}_{2} \mathrm{~B}_{2}$ can be explained by the relieve of the lattice stresses generated by the off-stoichiometry of the $M_{23}(C, B)_{6}$ and $\eta$ $\mathrm{Fe}_{3} \mathrm{Mo}_{3} \mathrm{C}$ carbides and the large amount of vacancies formed due to volume shrinking during crystallization. For instance stacking faults, partial dislocations and twining is observed with advanced crystallization in the $\mathrm{M}_{23}(\mathrm{C}, \mathrm{B})_{6}$ structure. According to the crystal configuration of this phase, with 92 metallic atoms per unit cell given by Goldsmidth [44], an approximate ratio up to 1:2 of $\mathrm{Fe}$ and $\mathrm{Cr}$, respectively, can be reached if only these two atoms are considered. The addition of substitutional Mo atoms, with a maximum allowed of 8 atoms per unit cell ( $\sim 7$ at.\%), would lead the formation of stoichiometric carbides of the form $(\mathrm{Fe}, \mathrm{Cr})_{21} \mathrm{Mo}_{2}(\mathrm{C}, \mathrm{B})_{6}$ with Fe being able to completely substitute $\mathrm{Cr}$ [30]. However, we found that this phase contains up to 10 at.\% of $\mathrm{Cr}$ at $1073 \mathrm{~K}$ [16] indicating that the equilibrium is not yet reached. As no more transformations occur until melting, the final equilibrium structure is composed of 71.7

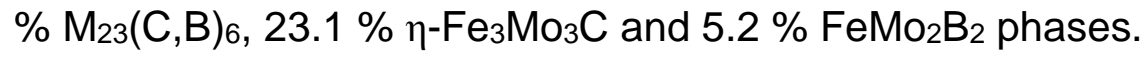

\section{Conclusions}


The metastable $\mathrm{Fe}_{50} \mathrm{Cr}_{15} \mathrm{Mo}_{14} \mathrm{C}_{15} \mathrm{~B}_{6}$ amorphous alloy reaches an equilibrium state through a complex transformation process that includes the formation of various nanosized metastable and stable phases during the devitrification process: $\alpha-\mathrm{Fe}, \chi-$ $\mathrm{Cr}_{6} \mathrm{Fe}_{18} \mathrm{Mo}_{5}, \mathrm{M}_{23}(\mathrm{C}, \mathrm{B})_{6}, \mathrm{M}_{7} \mathrm{C}_{3}, \mathrm{M}_{6} \mathrm{C}\left(\eta-\mathrm{Fe}_{3} \mathrm{Mo}_{3} \mathrm{C}\right)$ and $\mathrm{FeMo}_{2} \mathrm{~B}_{2}$, with $\mathrm{M}=\mathrm{Fe}, \mathrm{Cr}$, Mo. In this work, we used in-situ techniques of SR-XRD, TEM and DSC thermodynamic analyses to identify the forming phases, defined their stability range and provided information on their formation mechanism. Four exothermic peaks can be observed by DSC up to $1100 \mathrm{~K}$. The first peak corresponds to the parallel nucleation of $\alpha$-Fe and $\chi$ $\mathrm{Cr}_{6} \mathrm{Fe}_{18} \mathrm{Mo5}_{5}$, each one of these phases evolves independently and $\alpha$-Fe is only stable for a short interval at the beginning of the crystallization process. The formation of the main phase in the fully crystallized sample, $\mathrm{M}_{23}(\mathrm{C}, \mathrm{B})_{6}$, corresponds to the second peak, which accounts for more than $45 \%$ of the transformed phases. These primary transformations are controlled by diffusional processes with soft impingement. The formation of $\mathrm{M}_{7} \mathrm{C}_{3}$ is interface controlled, as indicated by its large Avrami exponent, and exhausts effectively the untransformed space. Besides the phases nucleated from the amorphous, the transformation to $\eta-\mathrm{Fe}_{3} \mathrm{Mo}_{3} \mathrm{C}$ occurs from the $\chi$ - $\mathrm{Cr}_{6} \mathrm{Fe}_{18} \mathrm{Mo} 5$ phase previously crystallized. At temperatures higher than $1050 \mathrm{~K}$, the final equilibrium

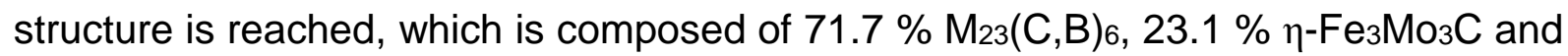
$5.2 \% \mathrm{FeMo}_{2} \mathrm{~B}_{2}$ phases.

\section{Acknowledgments}

We acknowledge T. Harzer and A. Szczepaniak for their great assistance in sample preparation for in-situ TEM measurements. D.C. acknowledges the support of MINECO (Grant FIS2014-54734-P) and Generalitat de Catalunya (Grant 2014SGR00581). Portions of this research were carried out at the light source Petra III at Desy, a member of the Helmholtz Associatioin (HGF). 


\section{References}

[1] C.A. Schuh, T.C. Hufnagel, U. Ramamurty, "Mechanical behavior of amorphous alloys", Acta Mater. 55 (2007) 4067-4109.

[2] S.V. Madge, "Toughness of bulk metallic glasses", Metals 5 (2015) 1279-1305.

[3] Y. Wei, X. Lei, L.S. Huo, W.H. Wang, A.L. Greer, "Towards more uniform deformation in metallic glasses: The role of Poisson's ratio", Mat. Sci. Eng. A 560 (2013) 510-517.

[4] J.J. Lewandowski, X.J. Gu, A. Shamimi Nouri, S.J. Poon, and G.J. Shiflet, "Tough Fe-based bulk metallic glasses", Appl. Phys. Lett. 92 (2008) 091918.

[5] M. F. Ashby, A. L. Greer, "Metallic glasses as structural materials", Scripta Mater. 54 (2006) 321-326.

[6] P.F. Gostin, A. Gebert, L. Schultz, "Comparison of the corrosion of bulk amorphous steel with conventional steel”, Corr. Sci. 52 (2010) 273-281.

[7] M.J. Duarte, J. Klemm, S.O. Klemm, K.J.J. Mayrhofer, M. Stratmann, S. Borodin, A.H. Romero, M. Madinehei, D. Crespo, J. Serrano, S.S.A. Gerstl, P.-Pa Choi, D. Raabe, F.U. Renner, "Element-resolved corrosion analysis of stainless-type glass-forming steels", Science 341 (2013) 372.

[8] W.H. Wang, C. Dong, C.H. Shek, "Bulk metallic glasses", Mat. Sci. Eng. R 44 (2004) 45-89.

[9] A. Inoue, A. Takeuchi, "Recent development and application products of bulk glassy alloys", Acta Mater. 59 (2011) 2243-2267.

[10]A. Inoue, F.L. Kong, "Bulk Metallic Glasses: Formation and Applications", Reference Module in Materials Science and Materials Engineering, 2016.

[11]A. Inoue, "Stabilization of metallic supercooled liquid and bulk amorphous alloys", Acta Mater. 48 (2000) 279-306.

[12]W.H. Wang, "Roles of minor additions in formation and properties of bulk metallic glasses", Prog. Mater. Sci. 52 (2007) 540-596.

[13]C. Suryanarayana, A. Inoue, "Iron-based bulk metallic glasses", Int. Mater. Rev. 58 (2013) 131-166.

[14]A. Inoue, B.L. Shen, H.M. Kimura, "Fundamental Properties and applications of Fe-based bulk glassy alloys", J. Metastable Nanocrys. Mater. 20-21 (2004) 3-12.

[15] R. Raghavan, V.V. Shastry, A. Kumar, T. Jayakumar, U. Ramamurty, "Toughness of as-cast and partially crystallized composites of a bulk metallic glass", Intermetallics 17 (2009) 835-839.

[16] M.J. Duarte, A. Kostka, J.A. Jimenez, P. Choi, J. Klemm, D. Crespo, D. Raabe, F.U. Renner, "Crystallization, phase evolution and corrosion of Fe-based metallic glasses: an atomic-scale structural and chemical characterization study", Acta Mater. 71 (2015) 20-30.

[17] C.C. Hays, C.P. Kim, and W. L. Johnson, " Microstructure controlled shear band pattern formation and enhanced plasticity of bulk metallic glasses containing in situ formed ductile phase dendrite dispersions", Phys. Rev. Lett. 84 (2000) 29012904. 
[18] S.F. Guo, L. Liu, N. Li, and Y. Li, "Fe-based bulk metallic glass matrix composite with large plasticity", Scripta Mater. 62 (2010) 329-332.

[19]S. F. Guo, K.C. Chan, and L. Liu, "Notch toughness of Fe-based bulk metallic glass and composites," J. Alloy Comp. 509 (2011) 9441-9446.

[20]H. Springer, R. Aparicio Fernandez, M.J. Duarte, A. Kostka, D. Raabe, "Microstructure refinement for high modulus in-situ metal matrix composite steels via controlled solidification of the system Fe-TiB2", Acta Mater. 96 (2015) 47.

[21]D.C. Hofmann, "Bulk metallic glasses and their composites: A brief history of diverging fields", J. Mater. 2013 (2013) 517904.

[22] K. Lu, "Nanocrystalline metals crystallized from amorphous solids: nanocrystallization, structure, and properties", Mat. Sci. Eng. R 16 (1996) 161221.

[23]M.E. McHenry, M.A. Willard, D.E. Laughlin, "Amorphous and nanocrystalline materials for applications as soft magnets," Prog. Mater. Sci. 44 (1999) 291-433.

[24]M.A. Meyers, A. Mishra, D.J. Benson, "Mechanical properties of nanocrystalline materials", Prog. Mater. Sci. 51 (2006) 427-556.

[25] V. Ponambalam, S.J. Poon, G.J. Shiflet, "Fe-based bulk metallic glasses with diameter thickness larger than one centimeter", J. Mater. Res. 19 (2004) 13201323.

[26] Z.P. Lu, C.T. Liu, W.D. Porter, "Role of yttrium in glass formation of Fe-based bulk metallic glasses", Appl. Phys. Lett. 83 (2003) 2581.

[27]F. Zhai, E. Pineda, M.J. Duarte, D. Crespo, "Role of niobium in glass formation of Fe-Cr-Mo-C-B-Nb bulk metallic glasses", J. Alloy Comp. 604 (2014) 157-163.

[28]D. Peckner, I.M. Bernstein, "Handbook of stainless steels", MCGraw-Hill, New York, 1977.

[29]P.-J. Cunat "Alloying Elements in Stainless Steel and Other ChromiumContaining Alloys", Euro Inox, Paris, 2004.

[30]A. Inoue, L. Arnberg, M. Oguchi, U. Backmark, N. Bäckström, T. Matsumoto, "Preparation of Fe-Cr-Mo-C amorphous powders and microstructure and mechanical properties of their hot-pressed products", Mat. Sci. Eng. 95 (1987) 101-114.

[31]A.-C. Dippel, H.-P. Liermann, J. T. Delitz, P. Walter, H. Schulte-Schrepping, O. H. Seeck, H. Franz, "Beamline P02.1 at PETRA III for high-resolution and highenergy powder diffraction", J. Synchrotron Rad. 22 (2015) 675-687.

[32] http://www.esrf.eu/computing/scientific/FIT2D/

[33] M. Duchamp, Q. Xu, R. Dunin-Borkowski, "Convenient preparation of high-quality specimens for annealing experiments in the transmission electron microscope", Microsc. Microanal. 20 (2014) 1638-1645.

[34]M.A. van Huis, N.P. Young, G. Pandraud, J.F. Creemer, D. Vanmaekelbergh, A.I. Kirkland, H.W. Zandbergen, "Atomic imaging of phase transitions and morphology transformations in nanocrystals", Adv. Mater. 21 (2009) 4992-4995.

[35]P.A. Stadelmann, JEMS - Electron Microscopy Software, Version 4.2231U2014, Copyright P. Stadelmann 1999-2014, JEMS-SAAS, Switzerland, 2014.

[36] M. Avrami, "Kinetics of Phase Change. I General Theory", J. Chem. Phys. 7 (1939) 1103-1112. 
[37] J. Farjas, P. Roura, "Modification of the Kolmogorov-Johnson-Mehl-Avrami rate equation for non-isothermal experiments and its analytical solution", Acta Mater. 54 (2006) 5573-5579.

[38] H.K.D.H. Bhadeshia, "Kinetics of simultaneous transformations", Japan Inst. Metals, Proceedings 12 (1999) 1445-1452.

[39]T. Pradell, D. Crespo, N. Clavaguera, M. T. Clavaguera-Mora, "Diffusion controlled grain growth in primary crystallization Avrami exponents revisited", J. Phys.: Condens. Matter 10 (1998) 3833-3844.

[40] M. T. Clavagzera-Mora, N. Clavaguera, D. Crespo, T. Pradell, "Crystallization kinetics and microstructure development in metallic systems", Prog. Mater. Sci. 47 (2002) 559-619.

[41] J. W. Christian, "The Theory of Transformations in Metals and Alloys", Pergamon Press, Oxford, $2^{\text {nd }}$. Edition 1981.

[42]D. V. Louzguine, A. Inoue, "Crystallization behavior of Ti50Ni25Cu25 amorphous alloy", J. Mater. Sci. 35 (2000) $4159-4164$.

[43] J. O. Anderson, N. Lange, "An experimental study and a thermodynamic evaluation of the Fe-Cr-Mo system", Metall. Trans. A 19 (1988) 1385.

[44] J. S. Kaspar, "The ordering of atoms in the chi-phase of the iron-chromiummolybdenum system", Acta Metall. 2 (1954) 456-461.

[45] H. E. Kissinger, "Reaction kinetics in differential thermal analysis", Anal. Chem. 29 (1957) 1702-1706.

[46] G. He, W. Löser, J. Eckert, "Devitrification and phase tansformation of (Ti0.5Cu0.25Ni0.15Sn0.05Zr0.05)100-xMox metallic glasses“, Scripta Mater. 50 (2004) 7-11. 


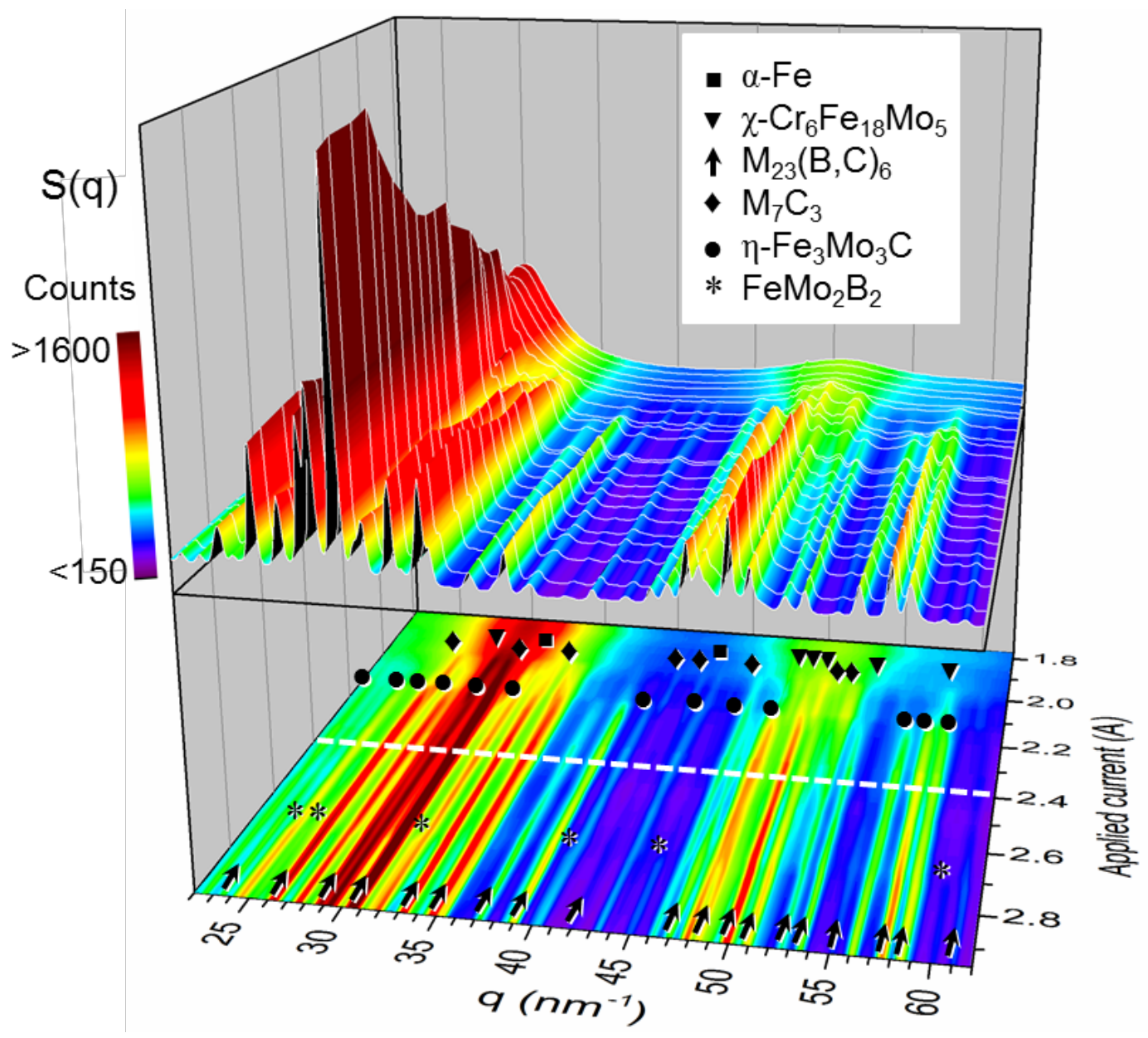

Fig. 1. Integrated in-situ XRD patterns and corresponding 2D projection acquired at a step rate of $\sim 0.05 \mathrm{~A} / \mathrm{min}$ showing the sequence of the appearing phases. The dashed white line indicates the current at which full crystallization is reached. 


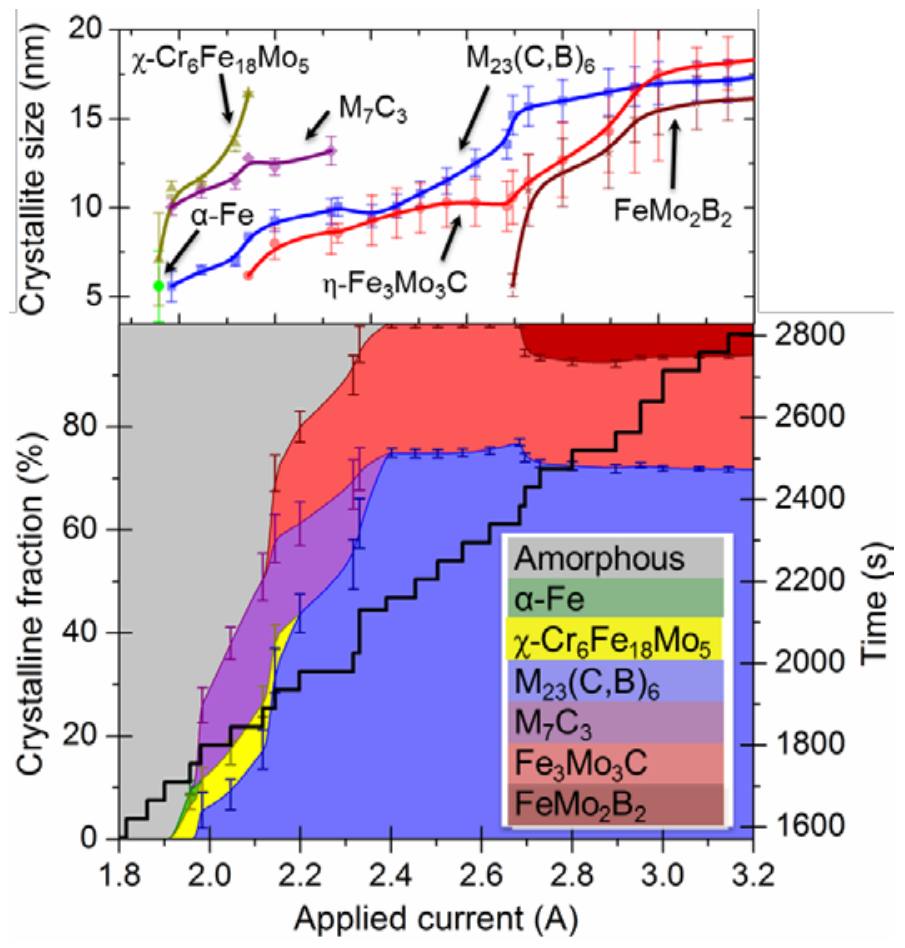

Fig. 2. Quantification of the crystalline fraction and crystallite size of the different phases by the Rietveld method. The black line in the lower plot indicates the manual step current increase followed during the measurement. 


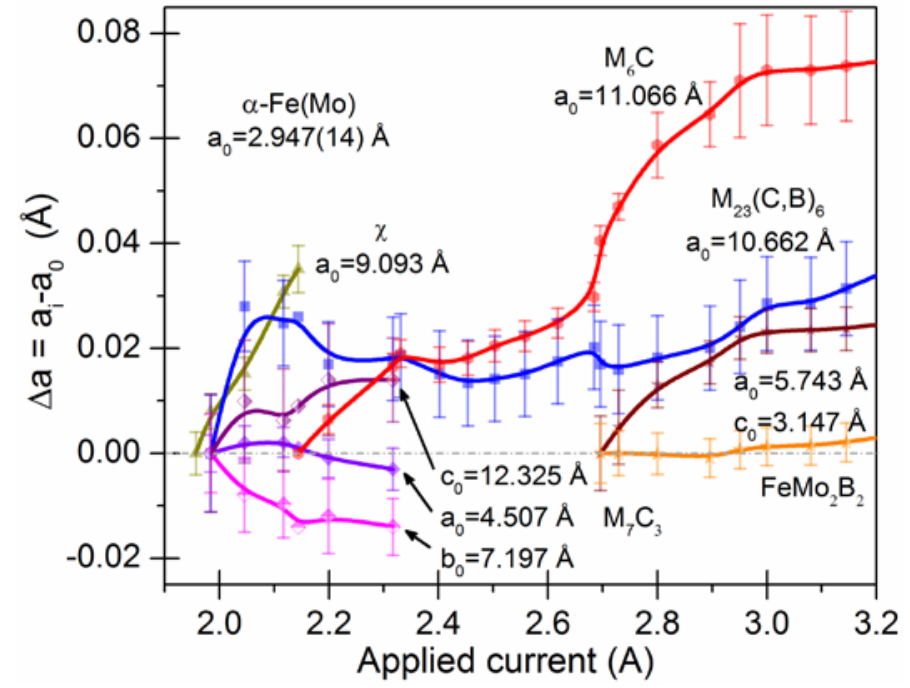

Fig. 3. Change in the lattice parameters with respect to the applied current. 

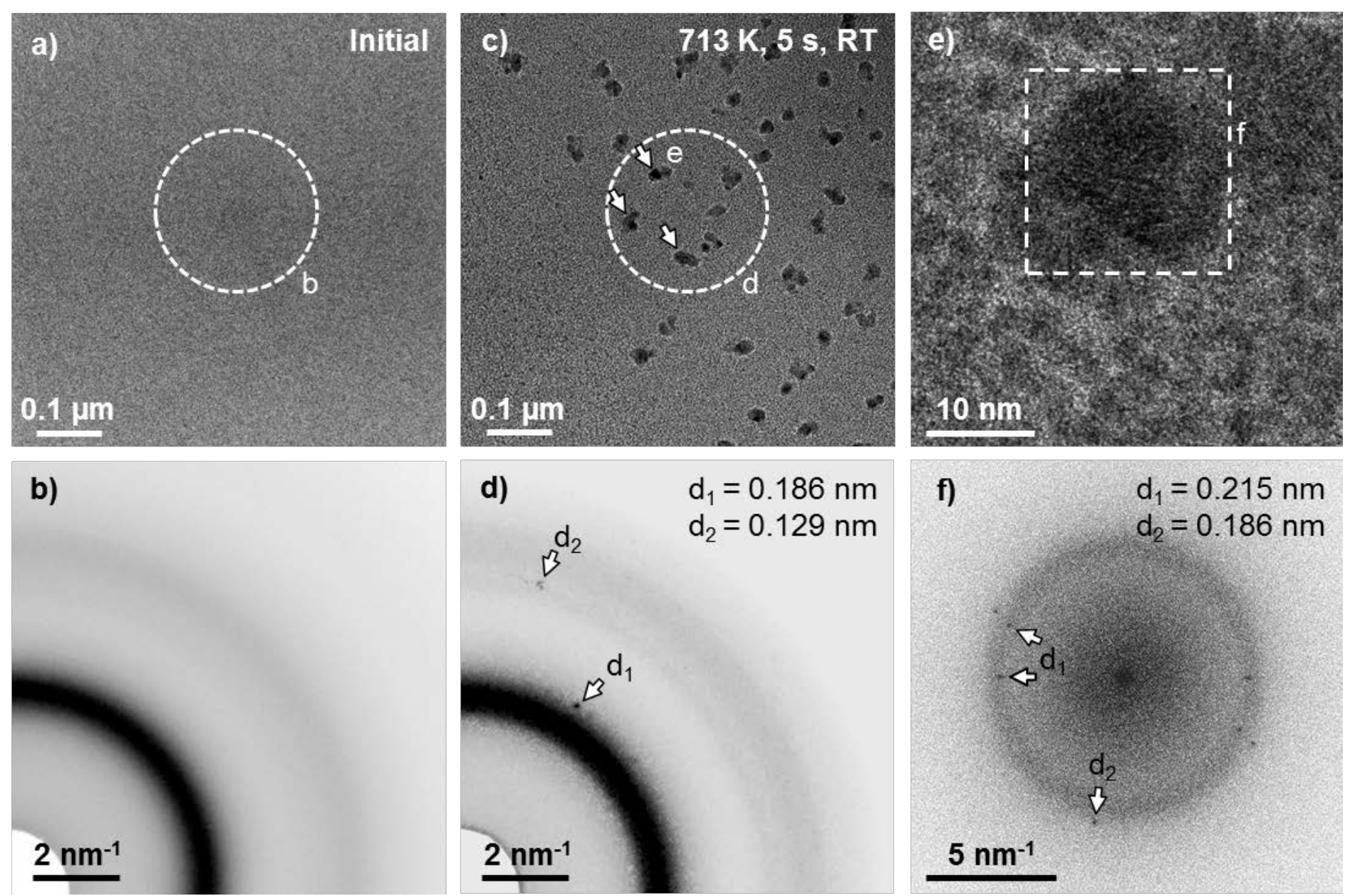

Fig. 4. Initial crystallization by in-situ TEM: (a) BF image and (b) SAD of the fully amorphous sample in the initial conditions; $(c, d)$ first crystallites at $713 \mathrm{~K}$ and $(e, f)$ HR-TEM image and corresponding FFT of one of the nucleated crystals indicated by arrows in (c). The dashed circles represent the aperture size used for the SAD acquisition. 
Fig. 5. In-situ TEM crystallization sequence during isothermal annealing at $723 \mathrm{~K}$ : (a-f) micrographs recorded after different annealing time; $(\mathrm{g}-\mathrm{i})$ diffraction patterns of the regions indicated by dashed circles; (j-I) corresponding radial intensity profile of the rotational averaged diffraction patterns in $\mathrm{g}$-i.
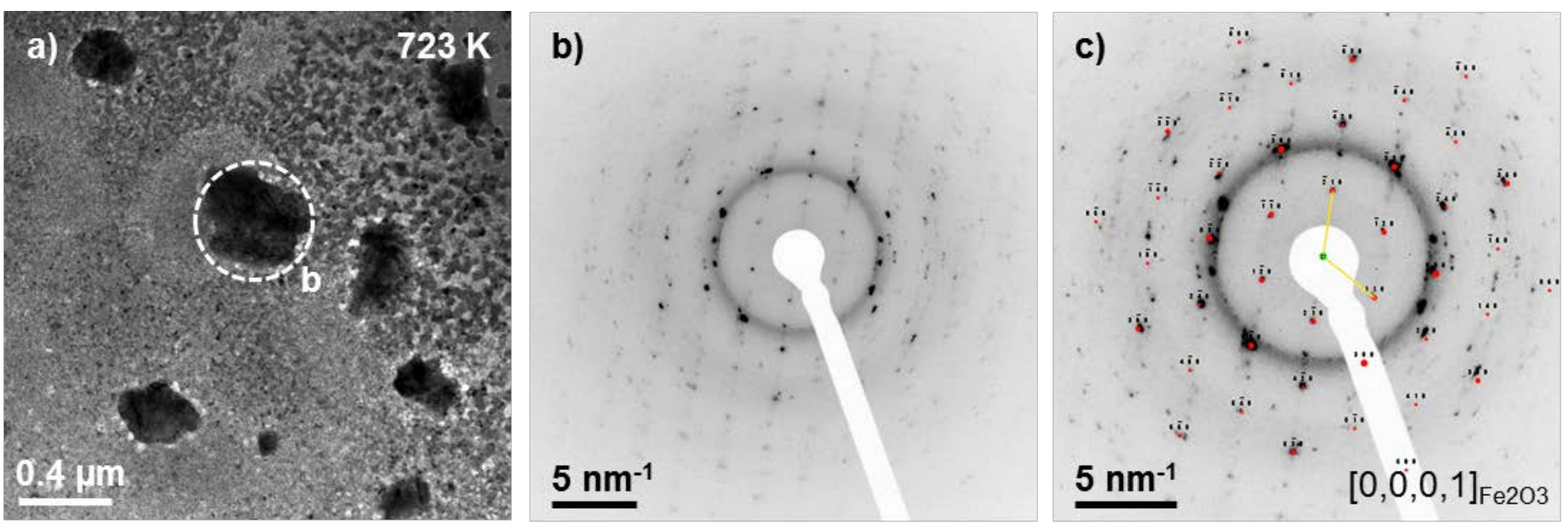

Fig. 6. Oxides formation: (a) BF image; (b) SAD pattern corresponding to the white dashed region in a); (c) overlay of the simulated $\mathrm{Fe}_{2} \mathrm{O}_{3}$ diffraction pattern (red dots). 

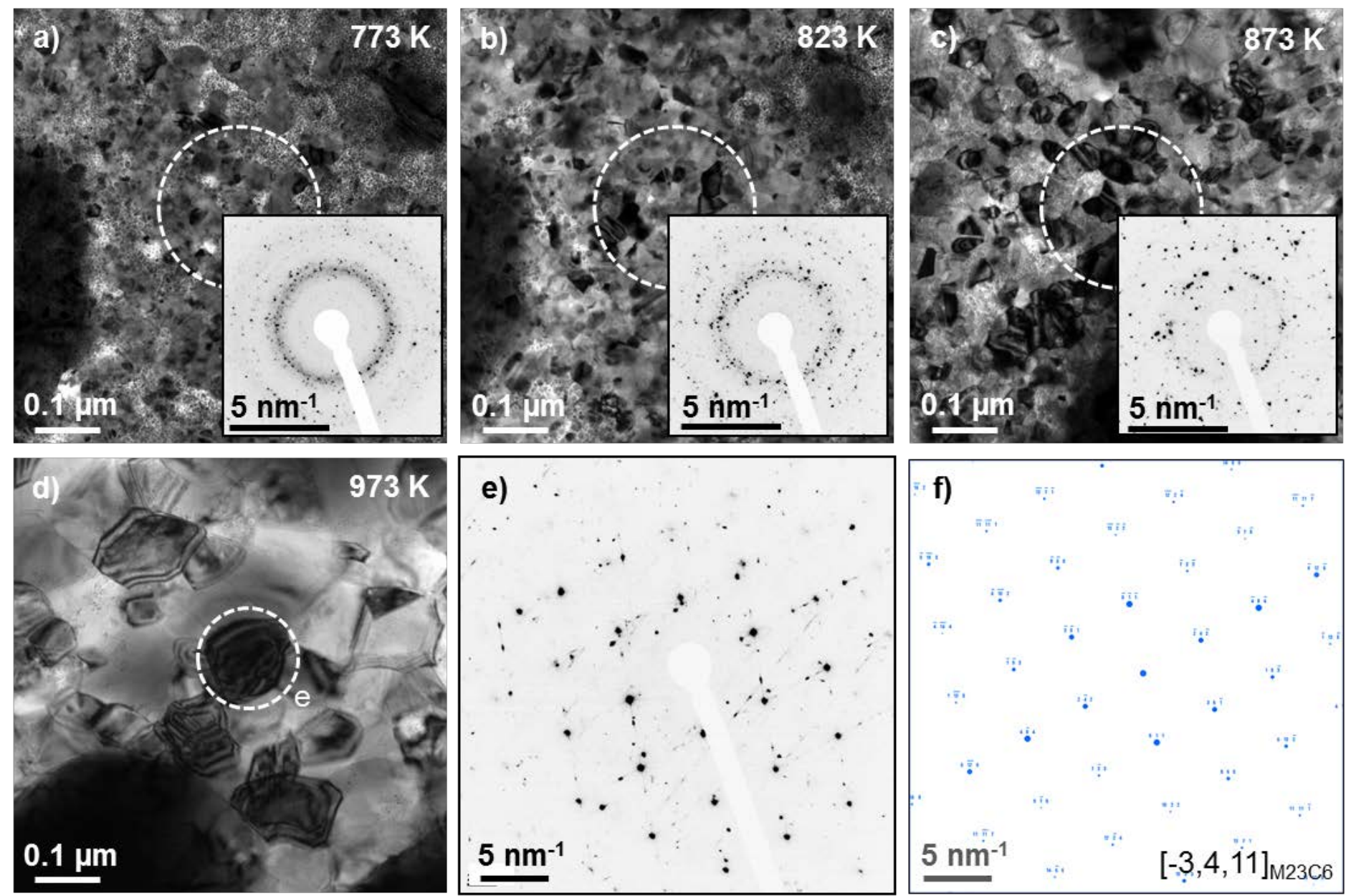

Fig. 7. Overview of the microstructure and SAD pattern taken from the corresponding white dashed region after heating at (a) $773 \mathrm{~K}$, (b) $823 \mathrm{~K}$ and (c) $873 \mathrm{~K}$. (d) Final microstructure after heating to $973 \mathrm{~K}$; (e) SAD pattern corresponding to the region encircled in d); (f) simulated diffraction pattern of $\mathrm{M}_{23} \mathrm{C}_{6}$. 


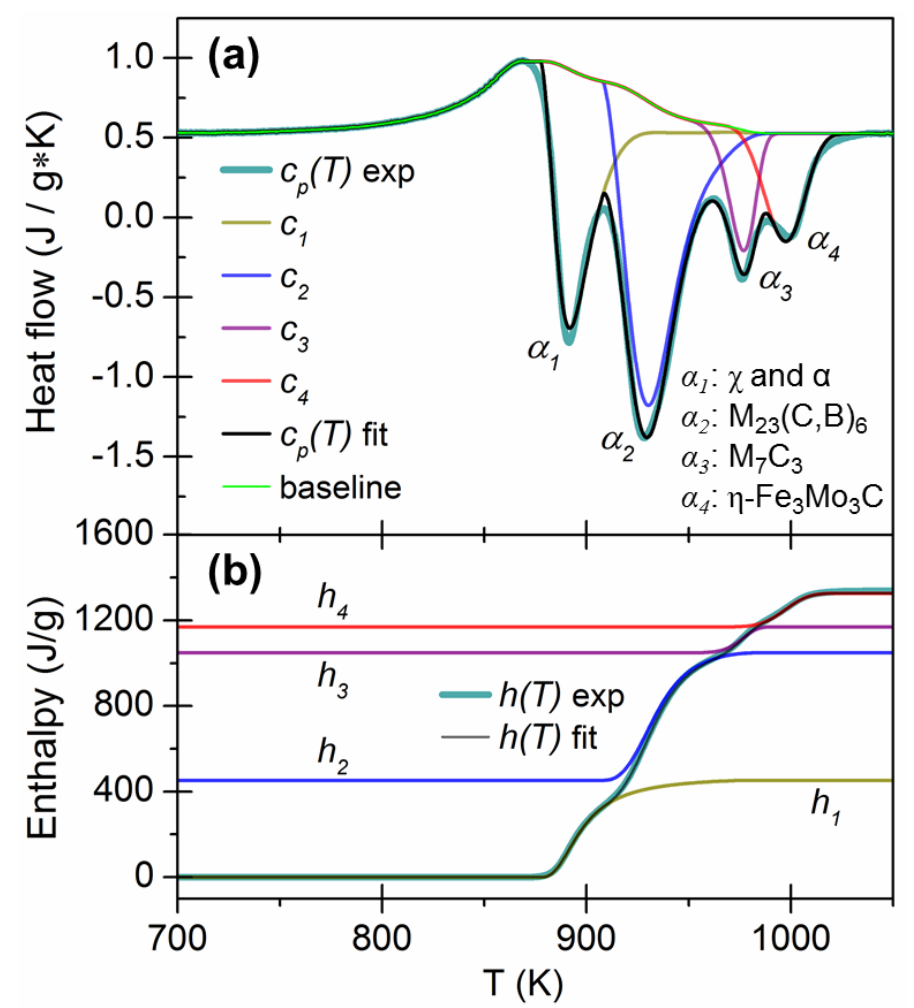

Fig. 8. (a) DSC curve and fitted data of the individual transformations. (b) Determined enthalpy as a function of the temperature. 